\title{
Context modelling for serendipitous discoveries in exploratory mobile search over social media
}

\author{
Juan David Millan Cifuentes \\ City University London \\ School of Informatics \\ Juan.Millan-Cifuentes.1@city.ac.uk
}

\begin{abstract}
Ubiquitous information seeking systems have changed the way urban citizens understand and explore their world. At one time our five senses were the only way we could comprehend our surroundings, but now smartphones and other devices with access to social media have become bodily extensions which allow humans to expand their social interaction and sense what is "really happening". This paper proposes research into serendipitous discoveries and casual mobile explorations enabled by context modelling over social media. This research will use the "surrounding context" concept as lens to present information and create aggregated views of what is happening with multiple visualization techniques like real-time ambient tag cloud and heat maps. The evaluation will follow a user-centred approached both in laboratory and naturalistic scenarios focussing on leisure.
\end{abstract}

Social Media, Serendipity, Context Modelling, Exploratory Mobile Search, Casual Search

\section{INTRODUCTION}

Information seeking systems such as search engines often perform well when users have a clear idea of their information need where the user search activity is limited to lookup or verify (Marchionini, 2006). But search is not just a findability problem, especially when users are seeking insightful knowledge as they explore (they want something, but they might not know how or where to look for it) or they don't know what they need (they may start with little or no purpose in mind and change it with another after a successful new discovery) (Spencer, 2006). However if this is a priority concern over web search design experience for a traditional desktop user, how much more so when the primary user is mobile, strongly affected by contextual factors (such as location, time, task or social group) and the search is motivated by strong casual desire instead of a knowledge gap (Wilson et al., 2010).

Moreover, humans are social creatures even when they search for information. Their casual desires could be motivated, satisfied and understood within a social context. This may explain why so many people visit social media sites. They want to share what is happening to them whilst at the same time sense what is trending around the world or an urban location where they plan to go. For example some people when travelling rather than to follow a guide book might prefer to go where "the locals go"

(Balduini et al., 2012). But commercial search engines fail to respond to complex contextual queries such as; which is the most popular pub nearby after the last performance in the theatre? or what is the most popular restaurant for university students to have lunch around the university campus? According to recent studies many people now prefer to ask this type of question in their Social Network Sites (SNS) because they may receive more personalized and trustworthy answers (Morris et al, 2010).

In this regard, the proposed research explores the role of context modelling and social media to enable serendipitous discoveries and exploration for mobile users performing leisure searches. Both in academia and industry, there has been much research on the social dimension of search using geo-related content especially from Twitter and Facebook streams to satisfy the information needs of mobile users (Church et al., 2010; Balduini et al., 2012; Oussalah et al., 2013). However, they have not focused on how to model the end user's context for social seeking information beyond the physical location (Göker \& Myrhaug, 2008; Emmanouilidis et al., 2013). Nor have they considered how to design for serendipity and exploration when a mobile user undertakes local searches motivated by spontaneous curiosity or casual leisure (Wilson et al., 2010). This research will propose an user-centred evaluation both in the 
laboratory and naturalistic scenarios using a prototype application which will crawl real time streams of data from social media and create aggregated views of what is happening based in the user's "surrounding context" when engaging in casual mobile search.

\section{RELATED WORK}

The following section focus on four related areas to underline this work: serendipity, context modelling, social media and exploratory mobile search.

\subsection{Serendipity}

This property "may indeed be the holy grail of the search experience" (Russell-Rose \& Tate, 2013: 77) and plenty of information seeking research has seen its value, but serendipity is a difficult property to study formally from an engineering perspective because it is hard to define and capture. For this research serendipity goes beyond the accidental discovery and explores "what it means to have a prepared mind and an infrastructure to support discovery" (André et al., 2009).

Common design search patterns such as "See Also" panels, recent searches and viewed items are used to support serendipitous exploration on commercial sites like Amazon and eBay. In the academic field, recent systems have been designed to promote serendipitous discoveries over web search (Erdelez \& Rioux, 2000). These have used standard information retrieval techniques to model interest based on previous digital experience such as email and chat archives, they then use this information to highlight terms or pages (Beale, 2007; Hangal et al., 2012). The aid provided by these systems enhances the mind capability to recall previous experience in the moment of searching and assumes that increasing the mind's ability of recall might fill the knowledge gap to explore and discover.

In this proposal, serendipity would be assumed as not just a personal act, but rather a social process where unexpected insights from other people's behaviours may help the individual make valuable decisions they would otherwise have been unable to make. Every invention or discovery has been understood in a social context even when chance has played a vital role. The opposite is also true and is well documented, there have been cases where due to some social context, individuals have lacked the capacity to understand the value of other people or their own discoveries even when they are not "accidental" (Kohn, 1989). To exemplify the importance of social context, think of a traveller who is looking for new places of interest, and then instead of following the plans of written tourist brochures or guides, chooses to unexpectedly change their destination based on key recommendations given by another traveller or local person (Makri \& Blandford, 2012). Certainly, social media as a ubiquitous communication channel could improve this kind of interaction.

\subsection{Context Modelling}

The idea of context may seem obvious when we reflect on the way we understand conversations or information, but it could be quite a challenge to apply it practically. Beside context as term has been misused. It has become for information retrieval and computer science domains almost a buzz word to pique the interest of an incautious reader.

There are several definitions. For Schilit (1994) context-aware computing defines context as "where you are, who you are with, and what resources are nearby". On the other hand, Morse (2000) context is explained as "implicit situational information"; Schmidt (1999) goes further and interprets context as "interrelated conditions in which something occurs" pointing out possible relations between context features. A more comprehensive definition of context is provided by Dey (2001) when he defined it as "any information that can be used to characterise the situation of an entity". All these definitions, although different assume context is associated with the user's surrounding rather than the user model that focuses on their inner states.

However these definitions don't give practical ways in which context could be used for an information seeking application. Therefore User Context Model frameworks like Myrhaug \& Goker (2002) are needed due to its extensiveness and logical division of the context universe in five categories (Task, Spatiotemporal, Personal, Social, and Environmental). This framework has been adopted for the purpose of modelling the user's context whilst performing casual mobile searches for information using social media. The main contribution regarding context modelling will be an empirical study in the relationship between context features, exploration and the user perception of an "unexpected insightful discovery".

\subsection{Social Media}

Media content creation, edition and distribution has been transformed by SNS and smart mobile devices like mobile phones, tablets and other ubiquitous sensors. World events are documented and captured in real-time by individuals and organizations (e.g. sport events, earthquakes, hurricanes, stock markets, etc.) (Teevan et al., 2011). Media content is generated at astonishing rates by heterogeneous multilingual social mass of people who just want to communicate their thoughts, opinions and values. 
Streams of media content are extremely important as sources of information. But most of their true value is still undiscovered due to complex technical data mining challenges such as inconsistent quality (e.g. photomontage, stemming text with misspelling or social slang), lack of data (e.g. few occurrence of the same information) and the dynamic nature (e.g. newer information makes older sources irrelevant, millions of messages every minute force index scalability).

On the other hand, social media search design experience continues to ignore the user's context in the collection and delivery of the content. In other words, social media search is context free. Meanwhile, the user's perception is limited, whereas the total information is almost unending.

Paradoxically, more than ever before media content posted online has spatio-temporal and social context metadata. For example every photo and message upload in the SNS has a creation date and it is created by someone connected with a social graph of friends or followers. In addition, plenty of media content is loaded with geoinformation due to inclusion of GPS and other location systems (Derczynski et al., 2013).

As previously mentioned, this research endeavours to use the "surrounding context" concept as lens to filter and present information from social media and create aggregated views of what is happening (Göker \& Myrhaug, 2002).

\subsection{Exploratory Mobile Search}

When looking for leisure activities a mobile user's information search goals could be:

- Show me some cool local venue I don't already know near my location

- Waiting for my girlfriend at the station. Searching for the latest information.

These goals highlights that mobile search is not just a findability problem. The search of mobile users is affected by strong contextual factors and triggered by the hunger for casual information or simple curiosity rather than a fixed idea of what they want or an explicit need (Wilson et al., 2010). Therefore it would involve more than lookup search mode because sometimes users might not even know where to start the search (Marchionini, 2006).

When users lack "knowing what they need to know" in order to find or discover they need to build up an overall view of the information landscape without restriction and discover new patterns which will broaden their perception and help them to articulate their information needs (Spencer, 2006).

Nonetheless most research into exploratory search has been carried out for desktop, not for mobile users (Church et al., 2010) and with specialized experts looking for new areas of research with good search skills rather than common users who may be trapped into a predetermined cognitive style (Marchionini, 2006; Kang et al., 2010). However mobile touchable devices are perfect search devices because their interfaces are not keyboard oriented, the mobile users must tap buttons, swiping or squeezing the screen with their fingers (Neumann \& Schmeier, 2012). This characteristic of mobile devices may facilitate users to be engaging in learn and investigative search mode with other visualization structures. For example the "more like this" design pattern for tablets transforms any search task into an enjoyable browsing experience inviting the user to explore and learn (Nudelman, 2010).

This research will explore context modelling and multiple visualizations as "infrastructure to support discovery" when the user's search are motivated by curiosity and casual leisure desire of experiencing new things (e.g. what is "really happening" around me that I don't already know?). It also will be more concerned with increasing recall than precision using multiple techniques of visualization such as a novel contextual ambient cloud tags (Baldauf et al., 2009) and heat maps instead of fixed menu layouts. Although ambient cloud tags (basically tag clouds of geo-related data filter by the users location) have been used before with different SNS like Flickr and Wikipedia based on the tags and geographic information of an article or photo, none have used real-time streams from social media to build them using different contextual features proposed by this research (Joshi et al., 2010; Baldauf \& Simon, 2010)

\section{MOTIVATION AND RESEARCH QUESTIONS}

The following scenario illustrates the goal of the research and also highlights the current problems with information services when users are in exploratory mode.

John is PhD Student at City University London. This is his third year and he is writing the results of his investigation. After he finishes writing, no one is in the office, it's already dark. He needs to relax, and have fun in a good place. He wants a beer. Before he used different applications to discover new places, but he finds most of them recommend the same places he has already been to and although the beer is good some places are not as cool as he would like even when they appear highly ranked. He wishes to broaden his knowledge of what is happening around him in order to make a good decision.

Now he opens the prototype application using his tablet. An ambient tag cloud is generated using social media information filtered by his surrounding context. The word "King" appears in big bold letters 
and it is growing in size. The tag clouds dynamically change as new information arrives from social media. After observing for a few seconds, he clicks "King" and then he receives a group of context related tweets from people going to the King's Head Theatre 5 blocks away from the university. He keeps reading the tweets, and he realizes that a lot of people seem interested. Even people he follows have commented about the performance. He also notices there are still some tickets because one of the resellers just sent a message using the social network. Instead of going to a Pub, he decides to change his destination and runs in order to arrive for the performance.

As this hypothetical scenario highlights, sometimes human beings want to discover new things and escape from the routine, often they don't know where to start and they find themselves exploring with no specific purpose. This research will aim to answer the following research questions or topics.

\begin{abstract}
Understand the role of serendipity, exploratory mobile search and user's context model in social media. What is the role of user context modelling in the serendipitous discovery and exploration of social media information? Which context features trigger more exploration or serendipity?
\end{abstract}

Effectiveness of social media content for serendipitous discoveries and exploration using context modelling in casual mobile search. Does context relevant social media affect serendipitous discovery and exploration while mobile users undertake casual search? Do contextual based visualizations such as ambient cloud tags and heat maps enable exploration and serendipitous discoveries in a casual mobile search over social media? Which context feature and visualization produces more engagement and satisfaction in a casual mobile search over social media?

\section{DESIGN ARCHITECTURE}

To the best of the author's knowledge, the only system in the academic field similar to this research has been the BOTTARI system (2012). The system merges Twitter streams and Points of Interest (POls) to recommend restaurants and other venues based on temporally weighted opinions. However it doesn't focus on the exploratory search behaviour and limits the user's context model to spatial features such as location. Furthermore, it doesn't explore the occurrence of serendipity. A recent beta commercial application called Spindle, which proposes a discovery social engine based on social media information from different social networks (Olanoff D., 2012).
In order to elaborate, an interesting empirical study regarding context features, exploration and serendipity, this research proposes a system to support contextual casual search over social media. The architecture proposes three main components based on current real time big data technologies (e.g. Storm ${ }^{1}$ ) and HTML5. The following figure depicts the high level architecture.
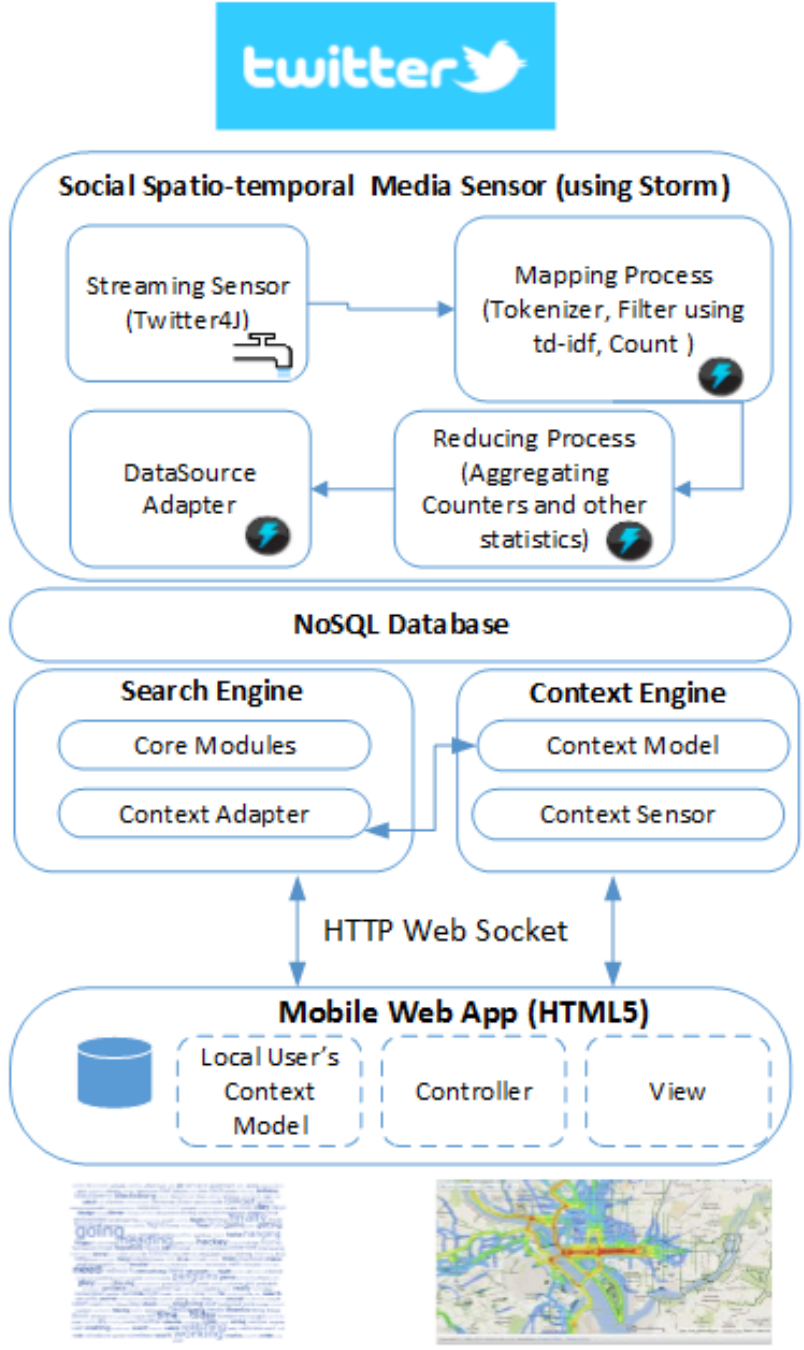

Figure 1: Prototype High-level Architecture Design to support exploratory mobile search and context modelling over spatiotemporal social media.

The first component is the Social Spatio-Temporal Sensor which will process social data (e.g. Twitter, Wikipedia, etc.), stored and indexed them based on content and spatiotemporal context. The tweets without geo-location would be clustered around the most relevant POls existing in the database. The second component is the Context Engine based on Myrhaug \& Goker (2002). The user's context model would include for the social media domain features such as time, location, preference (hashtags) and social relationships from SNS. The third component represents any search engine and the adapter to

\footnotetext{
${ }^{1}$ Twitter storm. Available https://github.com/nathanmarz/storm.
} 
the Context Engine. The fourth and final component is the mobile web application which is design around HTML5 standards (e.g. Geolocation, Websockets, etc.).

\section{EVALUATION AND EXPERIMENT}

In order to answer the previous research questions, the evaluation and experiment will follow an usercentred approach both in laboratory and naturalistic scenarios (Kelly, 2009).

For laboratory, simulated work task scenarios would be created in order to generate an informative environment for participants and help them with their judgments (Borlund, 2003). But accordingly to (Wilson et al., 2010), the simulated scenarios of casual search should also provide a "hedonistically motivated tasks" background. The scenarios will be created from social media streams of data (e.g. Twitter Streaming API filter by location and name of places).

For the naturalistic scenarios, a group of participants would use the application proposed in the previous section in their daily life while their interaction is logged. Also they would create a selfreport on the usage of the application.

After finishing both laboratory and naturalistic "experiments", interviews will be carried out for some of the participants.

Regarding subjective measures in the evaluation, this research will look especially into O'Brien \& Tom (2008) framework to analyse the effect of context features (e.g. location, time, social relationships from the SNS), and visualization over exploration engagement.

\section{CONCLUSION}

This work is an initial phase of $\mathrm{PhD}$ research where the main goal is to understand how context features could affect the casual mobile search and influence the "unexpected insightfup" discovery of information using real-time social media streams of data and multiple visualization techniques. This study can lead us to a better understanding of serendipity and casual mobile search from IR perspective. This research also endeavours to highlight the importance of designing the search experience using multiple user's context features.

\section{ACKNOWLEDGEMENT}

Thanks to A. MacFarlane, A. Göker, H. Myrhaug and the anonymous reviews for their helpful comments.

\section{REFERENCES}

André, P.; m. c. schraefel; Teevan, J. \& Dumais, S. T. (2009), Discovery is never by chance: designing for (un)serendipity., in Nick BryanKinns; Mark D. Gross; Hilary Johnson; Jack Ox \& Ron Wakkary, ed., 'Creativity \& Cognition' , ACM, , pp. 305-314 .

Baldauf, M., Fröhlich, P., Reichl, P. (2009). The Ambient Tag Cloud: A New Concept for Topicdriven Mobile Urban Exploration. Proceedings of the $3^{\text {rd }}$ European Conference on Ambient Intelligence, Salzburg, Austria: Springer LNCS.

Baldauf, M. \& Simon, R. (2010), Getting context on the go: mobile urban exploration with ambient tag clouds., in Ross Purves; Paul Clough \& Christopher B. Jones, ed., 'GIR' , ACM.

Balduini, M., et al. (2012), BOTTARI: An augmented reality mobile application to deliver personalized and location-based recommendations by continuous analysis of social media streams , J. Web Sem. 16 , 33-41.

Beale, R. (2007), Supporting serendipity: Using ambient intelligence to augment user exploration for data mining and web browsing, Int. J. Hum.Comput. Stud. 65 (5) , 421-433.

Borlund, P. (2003). The IIR Evaluation Model: A Framework for Evaluation of Interactive Information Retrieval Systems. In Information Research, volume 8. number 3, paper no. 152.

Church, K, et al. (2010), SocialSearchBrowser: a novel mobile search and information discovery tool., in Charles Rich; Qiang Yang; Marc Cavazza \& Michelle X. Zhou, ed., 'IUI' , ACM, pp. 101-110.

Derczynski, L., Yang, B., \& Jensen, C. S. (2013). Towards Context-Aware Search and Analysis on Social Media Data. In Proceedings of the 16th Conference on Extending Database Technology (EDBT 2013).

Dey, A. K. (2001). Understanding and Using using multiple user's context features and Context. Personal and Ubiquitous Computing, 5(1):4-7.

Erdelez, S. \& Rioux, K. (2000), Sharing information encountered for others on the Web, New Review of Information Behaviour Research 1(1), 219233.

Emmanouilidis, C.; Koutsiamanis, R.-A. \& Tasidou, A. (2013), Mobile guides: Taxonomy of architectures, context awareness, technologies and applications, J. Network and Computer Applications 36 (1) , 103-125.

Göker, A. \& Myrhaug, H. I. (2002), User Context and Personalisation, in 'ECCBR Workshop on 
Case Based Reasoning and Personalisation, Aberdeen'.

Göker, A. \& Myrhaug, H. I. (2008), Evaluation of a mobile information system in context, Inf. Process. Manage. 44 (1) , 39-65.

Hangal, S.; Nagpal, A. \& Lam, M. S. (2012), Effective browsing and serendipitous discovery with an experience-infused browser., in Carlos Duarte; Luís Carriço; Joaquim A. Jorge; Sharon L. Oviatt \& Daniel Gonçalves, ed., 'IUI' , ACM, pp. 149-158.

Joshi, D., Luo, J., Yu, J., Lei, P., Gallagher, A (2010) Rich location-driven tag cloud suggestions based on public, community, CMM'10, pp. 21 - 26

Kang, R.; Fu, W.-T. \& Kannampallil, T. G. (2010), Exploiting knowledge-in-the-head and knowledge-in-the-social-web: effects of domain expertise on exploratory search in individual and social search environments., in Elizabeth D. Mynatt; Don Schoner; Geraldine Fitzpatrick; Scott E. Hudson; W. Keith Edwards \& Tom Rodden, ed., 'CHI' , ACM, pp. 393-402 .

Kelly, D. (2009). Methods for evaluating interactive information retrieval systems with users. Foundations and Trends in Information Retrieval, 3(1-2), 1-224.

Kohn, A (1989). Fortune or failure: missed opportunities and chance discoveries. Cambridge, MA: Blackwell.

Makri, S. \& Blandford, A. (2012). Coming Across Information Serendipitously: Part 2: A Classification Framework. Journal of Documentation, 68(5), 706-724.

Marchionini, G. (2006), Exploratory search: from finding to understanding, Commun. ACM 49 (4), 41--46.

Morris M. R., et al. (2010). A Comparison of Information Seeking Using Search Engines and Social Networks. In Proceedings of 4th International AAAI Conference on Weblogs and Social Media, pp. 291-294.

Morse, D. R., Armstrong, S., and Dey, A. K. (2000). The what, who, where, when, why and how of context-awareness. In Conference on Human Factors in Computing Systems $(\mathrm{CHI})$, pages 371-371, The Hague, The Netherlands. ACM Press.

Neumann, G. \& Schmeier, S. (2012), Exploratory Search on the Mobile Web., in Joaquim Filipe \& Ana L. N. Fred, ed., 'ICAART (1)' , SciTePress, pp. 82-91.

Nudelman, G (2010), More Like This: A Design Pattern, [Online], Available: http://www.uxmatt ers.com/mt/archives/2010/01/more-like-this-adesign-pattern.php [12 May 2012].

O'Brien, H.L., Toms, E.G. (2008). What is user engagement? A conceptual framework for defining user engagement with technology. Journal of the American Society for Information Science and Technology 59(6), 938-955.

Olanoff, D. (2012). Latest Spindle For iOS Hits The Store: Discovery That's Great For Businesses And Consumers, Raises \$2.3M, [Online], Available: http://techcrunch.com/2012/11/15/thenext-version-of-spindle-for-ios-hits-the-storeand-is-an-amazing-promo-tool-for-businesses/ [05 May 2012].

Oussalah, M.; Bhat, F.; Challis, K. \& Schnier, T. (2013), A software architecture for Twitter collection, search and geolocation services, Knowl.-Based Syst. 37, 105-120.

Russell-Rose, T., Tate, T. (2013), Designing the Search Experience, 1st Edition, Morgan Kaufmann, Wattham.

Schilit, B. N.; Adams, N. \& Want, R. (1994), Context-Aware Computing Applications, in 'In Proceedings of the Workshop on Mobile Computing Systems and Applications', pp. 85-90 .

Schmidt, A., Beigl, M., and Gelersen, H.-W. (1999). There is More to Context than Location. Computers \& Graphics Journal, 23(6):893-902

Spencer, D. 2006. "Four Modes of Seeking Information and How to Design for Them". Boxes \& Arrows:_http://www.boxesandarrows.com/view /four_modes_of_seeking_information_and_how_ to design_for_them [05 May 2012].

Teevan, J., Ramage, D., \& Morris, M. R. (2011, February). \# TwitterSearch: a comparison of microblog search and web search. In Proceedings of the fourth ACM international conference on Web search and data mining. ACM. pp. 35-44.

Wilson, M. L. \& Elsweiler, D. (2010) Casual-leisure Searching: the Exploratory Search scenarios that break our current models. In: 4th International Workshop on Human-Computer Interaction and Information Retrieval, pp. 28-31. 